\title{
LES SONDAGES RADIOACOUSTIQUES DE L'ATMOSPHÈRE
}

\author{
Georges Dubosclard, Tayeb Ouazzani Touhami, \\ Jacques Fournet-Fayard et Yves B. Pointin \\ Laboratoire de météorologie physique \\ Observatoire de physique du globe de Clermont-Ferrand \\ Université Blaise Pascal \\ 12, avenue des Landais \\ 63000 Clermont-Ferrand
}

RÉSUMÉ

Le Rass (Radio Acoustic Sounding System) est un système de télédétection destiné à mesurer les profils de la température virtuelle. Il se compose d'un émetteur acoustique de forte puissance et d'un radar Doppler. La température virtuelle est déduite de la mesure de la vitesse de propagation du son dans l'air. Durant ces dernières années, les performances du Rass (et particulièrement sa portée verticale) se sont considérablement améliorées grâce à l'utilisation de radars strato-troposphériques (ST). Cet article se propose de faire le point sur la technique Rass. Nous insistons plus particulièrement sur les problèmes de physique de la mesure ainsi que sur leurs implications opérationnelles. À titre d'illustration, nous présentons les premiers résultats de sondages Rass effectués à l'aide d'un des radars ST du réseau de recherche Insu - Météo-France.

\section{ABSTRACT The radio acoustic sounding of the atmosphere}

The Radio Acoustic Sounding System (RASS) is a ground based remote sensing system for the measurement of virtual temperature profiles. It combines a high power acoustic transmitter with a Doppler radar, and virtual temperature is deduced from measurements of the speed of sound. The use of strato-tropospheric (ST) radars in recent years has dramatically increased RASS overal performance (particularly the height coverage). This paper is an overview of the status of RASS techniques with special emphasis on measurement physics and operational implications. As an example, we present the first results of RASS measurements carried out with one of the ST radars of the INSU - Météo-France research network.

À la suite de développements techniques et instrumentaux intervenus depuis près de vingt ans, les météorologistes disposent désormais, avec le profileur de vent, d'un sondeur capable de mesurer le vent en altitude avec une très bonne résolution spatiale et temporelle (Klaus et al., 1994). Néanmoins, la mesure simultanée et avec les mêmes performances d'autres paramètres atmosphériques est indispensable. Cela implique que l'effort de développement méthodologique et instrumental soit poursuivi. De ce point de vue, les résultats obtenus ces dernières années dans la mesure des profils de la température virtuelle à l'aide de sondages radioacoustiques sont particulièrement encourageants.

L'idée d'une utilisation conjointe des ondes acoustiques et radioélectriques pour mesurer les profils verticaux de la température de l'air a été envisagée au début des années soixante (Fetter, 1961 ; Atlas, 1962). Elle repose sur le fait que la vitesse de propagation du son dans l'air est proportionnelle à la racine carrée de la température virtuelle $T_{\mathrm{v}}$. 


\section{La température virtuelle}

Pour une particule atmosphérique d'air humide de température $T$ et de rapport de mélange $r$, la température virtuelle $T_{v}$ est définie comme la température d'une particule d'air sec de même masse volumique et de même pression, l'air sec et I'air humide étant considérés comme des gaz parfaits.

On peut calculer $T_{v}$ à partir de $T$ et de $r$ :

$\mathrm{T}_{\mathrm{v}}=\frac{1+1,608 \mathrm{r}}{1+\mathrm{r}} \mathrm{T}$

Le rapport de mélange $r$, qui représente la masse de vapeur d'eau associée à l'unité de masse d'air sec, est en général inférieur à 0,01 . L'écart $T_{v}-T$ est donc de l'ordre du degré, les valeurs les plus fortes étant atteintes dans les régions chaudes et humides de l'atmosphère.

Pour obtenir le profil vertical de $\mathrm{T}_{\mathrm{v}}$, il suffit donc d'envoyer dans l'atmosphère une onde sonore de forte puissance et d'en mesurer la vitesse de propagation à l'aide d'un radar Doppler.

Si la mesure repose sur un principe simple, sa mise en pratique a connu bien des vicissitudes. En 1965, les premiers essais réalisés par Allen et Weiner à l'aide d'un sondeur Emac (Electro Magnetic Acoustic Probe) mirent en évidence le bien-fondé de la méthode, mais ne permirent pas d'obtenir une mesure fiable de la température. Aussi, la technique fut-elle assez vite abandonnée. Une dizaine d'années plus tard, parallèlement au développement des premiers sodars, le problème de la mesure de la température est de nouveau considéré. Il aboutit à la mise au point d'un sondeur qui sera désormais connu sous le nom de Rass (Radio Acoustic Sounding System). À l'inverse de son prédécesseur, ce système fournit des mesures satisfaisantes de $T_{v}$ (Marshall, 1970 ; Frankel et Peterson, 1976). En même temps que les études expérimentales, l'approche théorique de la mesure s'approfondit, notamment grâce aux travaux de Clifford et Wang (1977), Clifford et al. (1978), Kon et Tatarskii (1980). Néanmoins, passée une phase de développements importants, le Rass va être, sinon abandonné, du moins mis en sommeil durant plusieurs années. Cette perte d'intérêt s'explique probablement par les performances modestes des systèmes réalisés, notamment en ce qui concerne la portée maximale qui ne dépasse pas, dans le meilleur des cas, un ou deux kilomètres. La cause de ces limitations est de nature essentiellement technologique : les radars utilisés, de conception relativement sommaire, émettaient des puissances faibles à l'aide d'antennes peu directives (voir tableau 1).

\begin{tabular}{|l|c|}
\hline Gamme de fréquences radar $(\mathrm{MHz})$ & $45 \rightarrow 150$ \\
\hline Angle d'ouverture du faisceau radar $\left(^{\circ}\right)$ & 30 \\
\hline Puissance radar $(\mathrm{W})$ & 30 \\
\hline Puissance acoustique (W) & 20 \\
\hline Portée maximale (km) & $1 \rightarrow 2$ \\
\hline
\end{tabular}

Tableau 1 - Caractéristiques des Rass de première génération.

Au début des années quatre-vingt-dix, à la suite du développement des radars stratotroposphériques ou radars ST (Klaus et al., 1994), les sondages Rass connaissent un regain d'intérêt. Les travaux menés aux États-Unis et au Japon (Angevine et al., 1994 ; Moran et al., 1991 ; Strauch et al., 1989 ; Tsuda et al., 1989) montrent que les performances de ces Rass de seconde génération s'avèrent bien supérieures à celles de leurs prédécesseurs. De plus, l'utilisation de plusieurs fréquences radioélectriques permet de couvrir une gamme d'altitudes étendue (voir tableau 2) tout en conservant une bonne précision (de l'ordre de $0,5 \mathrm{~K}$ ) sur la mesure de la température virtuelle. Ces améliorations spectaculaires font qu'à l'heure actuelle de nombreux radars ST sont équipés d'un Rass. Dans cet article, qui se propose de faire le point sur ce moyen de télédétection active de l'atmosphère, nous insistons plus particulièrement sur la physique de la 


\section{Radar, sodar, profileur de vent}

Le radar, le sodar et le profileur de vent sont des instruments destinés à l'exploration à distance (ou télédétection) du milieu atmosphérique. Leur principe de fonctionnement est analogue : un émetteur envoie dans l'atmosphère une onde, sous la forme d'une impulsion très brève ; cette onde est diffusée dans l'air par les hétérogénéités spatiales de l'indice de réfraction correspondant à la longueur d'onde $\lambda$ émise ; le récepteur capte la partie rétrodiffusée de l'onde.

On mesure l'intensité de l'onde rétrodiffusée en fonction du temps, avec un pas de temps $\tau$. Si c est la vitesse de propagation de l'onde dans l'atmosphère, cette analyse temporelle conduit à une mesure résolue en distance, avec une porte d'analyse (ou résolution en distance) de longueur $c \tau / 2$. Comme il y a émission d'une onde dans l'atmosphère, on parle d'instruments de télédétection active.

La mesure de l'intensité rétrodiffusée fournit la réflectivité du milieu diffusant. En version à effet Doppler, la mesure du décalage en fréquence de l'onde rétrodiffusée donne la vitesse radiale des cibles diffusantes.

Pour le radar détecteur de précipitations, l'onde émise est une onde électromagnétique centimétrique $(1 \mathrm{~cm}<\lambda<10 \mathrm{~cm})$. Les cibles diffusantes sont constituées par les gouttes de pluie. La porte d'analyse est de l'ordre de quelques kilomètres, la portée pouvant aller jusqu'à $200 \mathrm{~km}$.

Pour le sodar, l'onde émise est une onde acoustique $(10 \mathrm{~cm}<\lambda<30 \mathrm{~cm})$. Les cibles diffusantes sont constituées par les fluctuations d'indice liées à certaines formes de turbulence atmosphérique. En sondage vertical, le sodar permet de suivre la hauteur des couches d'inversion de température, avec une porte d'analyse de l'ordre de dix mètres et une portée d'un ou deux kilomètres. Avec plusieurs directions de sondage judicieusement choisies, le sodar Doppler permet de mesurer le profil du vent dans les basses couches de l'atmosphère.

Enfin, le profileur de vent (ou radar ST) fait appel à une onde électromagnétique métrique $(30 \mathrm{~cm}<\lambda<10 \mathrm{~m})$. Les cibles diffusantes sont constituées par les fluctuations d'indice liées à certaines formes de turbulence atmosphérique. Utilisé en version à effet Doppler, avec plusieurs faisceaux d'émission-réception judicieusement orientés, le profileur de vent permet de mesurer le profil de vent dans la troposphère et dans la stratosphère, avec une résolution verticale de quelques centaines de mètres.

mesure du Rass ainsi que sur ses implications pratiques. À titre d'illustration, nous présentons les premiers résultats de sondages Rass obtenus à l'aide d'un des radars VHF du réseau de recherche Insu - Météo-France, installé à l'observatoire de Physique du globe de Clermont-Ferrand (OPGC).

\begin{tabular}{|l|c|c|c|}
\hline Fréquence radar $(\mathrm{MHz})$ & 45 & 400 & 900 \\
\hline Angle d'ouverture du faisceau radar $\left(^{\circ}\right)$ & $2 \rightarrow 6$ & $4 \rightarrow 6$ & $2 \rightarrow 9$ \\
\hline Puissance radar $(\mathrm{W})$ & $10^{4}$ & $10^{2}$ & $10^{3}$ \\
\hline Puissance acoustique $(\mathrm{W})$ & 50 & 5 & 5 \\
\hline Portée $(\mathrm{km})$ & $2 \rightarrow 10$ & $0,4 \rightarrow 2,5$ & $0,2 \rightarrow 1,5$ \\
\hline
\end{tabular}

Tableau 2 - Caractéristiques des Rass de seconde génération.

PRINCIPE DU RASS CONDITION DE BRAGG

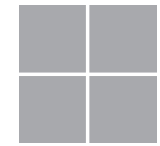

Comme nous l'avons indiqué, le principe de fonctionnement du Rass repose sur le fait que la vitesse du son dans l'air, $c_{a}$, est proportionnelle à la racine carrée de la température virtuelle $\mathrm{T}_{\mathrm{v}}$ :

$c_{\mathrm{a}}=20,047 \sqrt{\mathrm{T}_{\mathrm{v}}}$

$c_{a}$ étant exprimée en $\mathrm{m} \cdot \mathrm{s}^{-1}$. La mesure de la température virtuelle revient donc à celle de la vitesse de propagation du son.

La technique du Rass consiste à émettre dans l'atmosphère un signal sonore de forte puissance (généralement sous la forme d'une impulsion) qui, en se propageant, induit des fluctuations de l'indice de réfraction radioélectrique de l'air. Celles-ci agissent comme des cibles dont la vitesse de propagation (c'est-à-dire $c_{a}$ ) est mesurée à l'aide d'un radar Doppler. 


\section{Quelques relations fondamentales}

Une onde (monochromatique) qui se propage dans l'air est caractérisée par sa longueur d'onde $\lambda$, sa fréquence $f$ et son nombre d'onde $k$, avec $\mathrm{k}=1 / \lambda$

Si c est la vitesse de propagation de l'onde, on a

$\lambda=\mathrm{c} / \mathrm{f}$ et $\mathrm{k}=\mathrm{f} / \mathrm{c}$

Ainsi, pour une onde électromagnétique, $c=3.10^{8} \mathrm{~m} \cdot \mathrm{s}^{-1}$ (vitesse de la lumière).

- Si $f=3 \mathrm{GHz}, \lambda=10 \mathrm{~cm}$ (radar détecteur de précipitations).

- Si $f=30 \mathrm{MHz}, \lambda=10 \mathrm{~m}$ (radar ST VHF ou Very High Frequency).

- Si $f=600 \mathrm{MHz}, \lambda=50 \mathrm{~cm}$ (radar ST UHF ou Ultra High Frequency).

Pour une onde acoustique, c \# $300 \mathrm{~m} \cdot \mathrm{s}^{-1}$ (vitesse du son).

- Si $\mathrm{f}=1000 \mathrm{~Hz}, \lambda=30 \mathrm{~cm}$ (sodar).

\section{Effet Doppler}

Lorsque les cibles diffusantes se déplacent par rapport à l'émetteur-récepteur avec une vitesse radiale $v$, l'onde rétrodiffusée présente un décalage en fréquence $\Delta f=f . v / c$.

Ainsi, pour un radar $\mathrm{ST} V H F$, pour une vitesse radiale de $3 \mathrm{~m} . \mathrm{s}^{-1}$, on a $\Delta f / f=10^{-8}$, soit $\Delta f \# 0,3 \mathrm{~Hz}$.

Par analogie avec le cas du radar, l'équation du Rass désigne la relation exprimant la puissance $\mathrm{P}_{\mathrm{r}}$ de l'écho détecté à la distance $\mathrm{r}$ du radar en fonction des différents paramètres de fonctionnement du Rass (puissances acoustique et radioélectrique émises, $\mathrm{P}_{\mathrm{a}}$ et $\mathrm{P}_{\mathrm{e}}$; gain des antennes, $\mathrm{g}_{\mathrm{a}}$ et $\mathrm{g}_{\mathrm{e}}$ ). Une telle équation est établie en utilisant la théorie de la diffusion des ondes radioélectriques par des fluctuations d'indice (Tatarskii, 1971) et en supposant une atmosphère idéale, sans turbulence et sans vent. Dans le cas du Rass, les fluctuations de l'indice de réfraction radioélectrique sont engendrées par l'onde sonore lors de sa propagation dans l'atmosphère. Plusieurs équations du Rass ont été proposées (Marshall, 1970 ; Clifford et Wang, 1977 ; Bhatnagar et Peterson, 1979 ; Lataitis, 1992 ; Ouazzani Touhami, 1994). Toutes se ramènent à la forme générale :

$P_{r} \propto \frac{P_{a} g_{a} P_{e} g_{e}^{2} N^{2}}{r^{2}}\left\lfloor\frac{\sin \left|\left(2 k_{e}-k_{a}\right) \frac{N \lambda_{a}}{2}\right|}{\left(2 k_{e}-k_{a}\right) \frac{N \lambda_{a}}{2}}\right\rfloor$

où $\propto$ représente le symbole de proportionnalité.

Dans cette relation, $\mathrm{N}$ représente le nombre de périodes du signal acoustique contenues à l'intérieur d'une porte d'analyse (ou porte) du radar, $\mathrm{k}_{\mathrm{e}}$ et $\mathrm{k}_{\mathrm{a}}$ les nombres d'onde électrique et acoustique et $\lambda_{\mathrm{a}}$ la longueur d'onde acoustique. Il est à noter qu'une telle équation, contrairement à celle du radar, ne contient explicitement aucun terme caractérisant l'atmosphère. Son principal intérêt est, en fait, d'ordre pratique : la relation (2) permet, en effet, de définir les conditions de fonctionnement optimales d'un Rass. Ainsi, elle montre que la puissance reçue est proportionnelle au carré du nombre de sinusoïdes constituant le signal sonore. Or, à une fréquence a donnée, la durée d'émission du signal acoustique à l'intérieur d'une porte du radar s'écrit :

$\tau_{\mathrm{a}}=\frac{\mathrm{N}}{\mathrm{a}}$

L'intensité des échos du Rass est donc d'autant plus élevée que l'émission sonore est longue. Toutefois, nous montrerons plus loin que $\tau_{\mathrm{a}}$ possède une limite supérieure au-delà de laquelle la mesure de température n'est plus possible. 


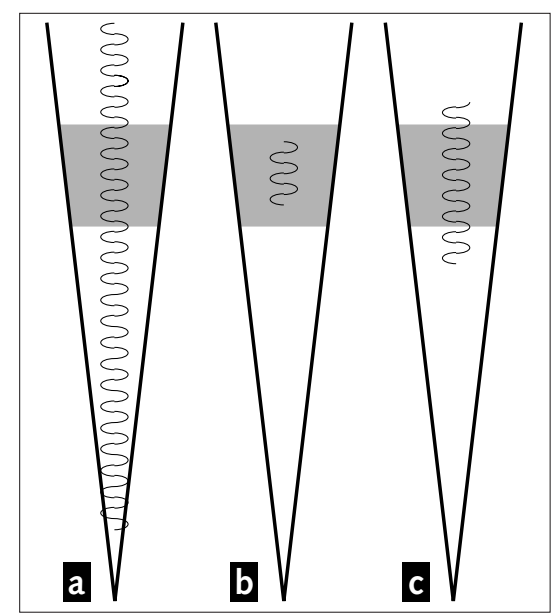

Figure 1 - Schémas illustrant les différentes possibilités d'émission du signal acoustique. (a) : émission continue ;

(b) : impulsion courte ;

(c) : impulsion longue. La partie grisée correspond à la porte d'analyse du radar.
La fréquence r du radar étant fixe, l'équation du Rass permet également de déterminer la fréquence acoustique produisant un écho dont l'intensité est maximale. L'équation (2) montre en effet que, toutes choses égales par ailleurs, le signal du Rass sera maximal lorsque :

$\mathrm{k}_{\mathrm{a}}=2 \mathrm{k}_{\mathrm{e}}$

Cette relation, dite condition de Bragg, peut également s'écrire sous la forme équivalente :

$\lambda_{\mathrm{a}}=\frac{1}{2} \lambda_{\mathrm{r}}$

où $\lambda_{\mathrm{r}}$ représente la longueur d'onde du radar. En utilisant (1), la fréquence acoustique de Bragg a pour expression :

$$
\text { в }=\frac{40,094 \sqrt{\mathrm{T}_{\mathrm{V}}}}{\lambda_{\mathrm{r}}}
$$

La condition de Bragg traduit le fait que les ondes radioélectriques, rétrodiffusées par les perturbations d'indice dues aux ondes sonores, sont en phase ou, en d'autres termes, que le signal du Rass est maximal, lorsque l'échelle spatiale de ces perturbations $\left(\lambda_{\mathrm{a}}\right)$ est égale à la demi-longueur d'onde du radar.

En utilisant la relation (5), la fréquence de Bragg correspondant à une fréquence du radar $r$ est donnée par :

$$
{ }_{\mathrm{B}}=2 \mathrm{r} \frac{\mathrm{c}_{\mathrm{a}}}{\mathrm{c}}
$$

Soit, si $\mathrm{c}_{\mathrm{a}} \approx 340 \mathrm{~m} \cdot \mathrm{s}^{-1}$ et $\mathrm{c}=3 \cdot 10^{8} \mathrm{~m} \cdot \mathrm{s}^{-1}$ :

$$
\text { В } \approx 2,27.10^{-6} \text { r }
$$

Pour un Rass utilisant un radar ST VHF ( $r \approx 45 \mathrm{MHz}$ ), la fréquence de Bragg se situe donc aux alentours de $100 \mathrm{~Hz}$ alors que dans le cas d'un radar UHF $\left({ }_{\mathrm{r}} \approx 1 \mathrm{GHz}\right)_{\text {B }}$ est de l'ordre de $2 \mathrm{kHz}$.

Pour cette discussion sur les performances du Rass, nous supposons que les

INFLUENCE DES CARACTÉRISTIQUES ACOUSTIQUES

Durée du signal acoustique émissions sonores et radioélectriques sont coaxiales.

À la suite des travaux de Peters et al. (1983), May et al. (1990) ainsi que ceux d'Adachi et al. (1992), il apparaît que la durée de l'émission sonore détermine, non seulement la puissance du signal reçu (conformément à l'équation du Rass), mais également la fréquence Doppler de l'écho Rass. En comparant l'extension spatiale du signal acoustique à la dimension d'une porte du radar, ces auteurs ont été conduits à distinguer trois cas : signal acoustique continu, impulsion acoustique courte et impulsion acoustique longue.

Si le signal acoustique est émis de façon continue (figure 1a), l'indice de réfraction radioélectrique, à l'intérieur d'une porte, fluctue à la fréquence de l'onde sonore. La fréquence Doppler de l'écho Rass est donc celle du signal acoustique émis ( ${ }_{\mathrm{a}}$ ) et la température virtuelle (proportionnelle au carré de la fréquence de Bragg ${ }_{\text {B }}$ ) ne peut pas être mesurée.

Dans le cas où le signal acoustique est émis sous la forme d'une impulsion de durée $\tau_{\mathrm{a}}$ son extension spatiale est égale à $\mathrm{c}_{\mathrm{a}} \tau_{\mathrm{a}}$. Lorsque cette dernière est inférieure à la dimension d'une porte du radar (figure $1 \mathrm{~b}$ ), la perturbation sonore constitue une cible isolée se déplaçant à l'intérieur de la porte à la vitesse $\mathrm{c}_{\mathrm{a}}$. La fréquence Doppler mesurée est alors égale à la fréquence de Bragg B. En utilisant (6) la température virtuelle est calculée à l'aide de la relation :

$\mathrm{T}_{\mathrm{V}}=6,221 \cdot 10^{-4}\left({ }_{\mathrm{B}} \lambda_{\mathrm{r}}\right)^{2}$

Le troisième cas à envisager est celui de l'impulsion acoustique longue, pour lequel l'étendue spatiale du signal sonore est supérieure à la dimension d'une porte du radar (figure 1c). May et al. (1990) ont montré que le spectre Doppler comporte 

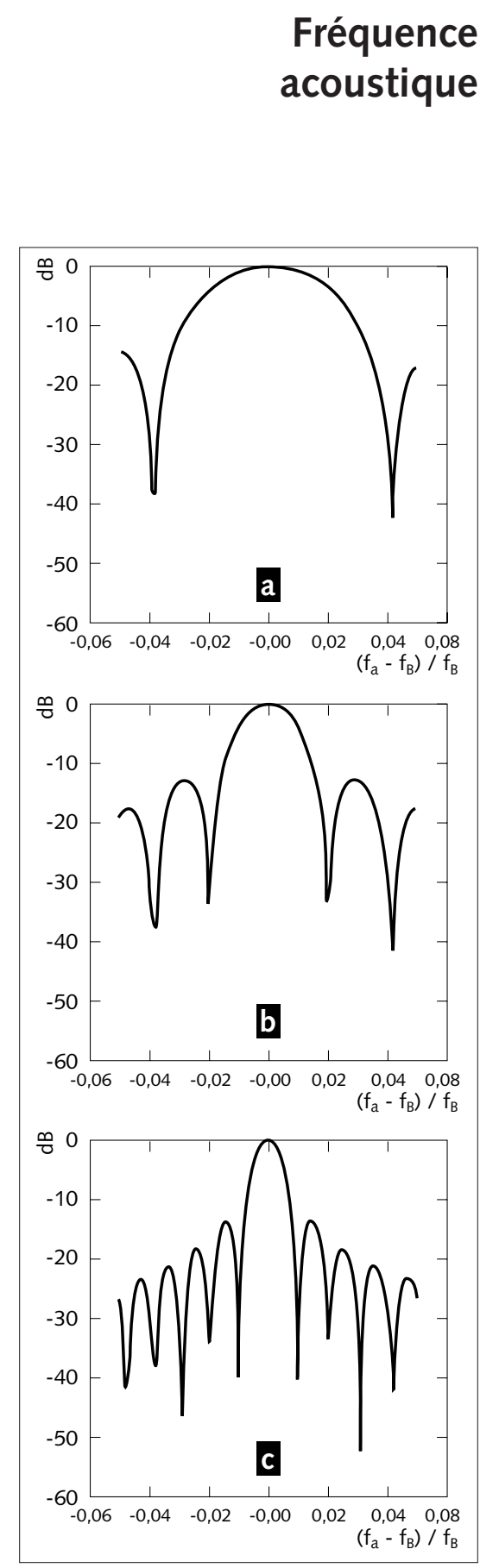

alors deux composantes : l'une à la fréquence de Bragg, , , et l'autre à la fréquence sonore, a. Cette situation peut, en effet, être considérée comme une combinaison des deux précédentes. Lorsque la perturbation sonore entre (ou sort) de l'espace occupé par une porte du radar, elle remplit partiellement cette dernière comme le fait une impulsion courte ; la fréquence mesurée est alors ${ }_{\text {B }}$. Pour une position intermédiaire de l'impulsion, l'extension spatiale du signal acoustique est supérieure à la dimension de la porte et les conditions sont alors équivalentes à celles d'une émission sonore continue pour laquelle la fréquence sonore a est mesurée.

En définitive, si $\tau$ désigne la période d'échantillonnage du signal reçu par le radar, la fréquence de Bragg est mesurée sans ambiguité si :

$\mathrm{c}_{\mathrm{a}} \tau_{\mathrm{a}}<\frac{\mathrm{c \tau}}{2}$

Ainsi, en prenant $\tau=2,5 \mu \mathrm{s}$, la durée $\tau_{\mathrm{a}}$ du signal acoustique doit être inférieure à environ $1 \mathrm{~s}$.

L'équation du Rass montre que la puissance de l'écho dépend de la fréquence sonore émise a par l'intermédiaire du terme :

$\left\lfloor\frac{\sin \left[\left(2 \mathrm{k}_{\mathrm{e}}-\mathrm{k}_{\mathrm{a}}\right) \frac{\mathrm{N} \lambda_{\mathrm{a}}}{2}\right]}{\left(2 \mathrm{k}_{\mathrm{e}}-\mathrm{k}_{\mathrm{a}}\right) \frac{\mathrm{N} \lambda_{\mathrm{a}}}{2}}\right\rfloor^{2}$

La figure 2, qui représente, pour différentes valeurs de $\mathrm{N}$, les variations de l'expression (9) en fonction de l'écart relatif par rapport à la fréquence de Bragg, montre que plus l'émission acoustique est longue (valeur élevée de $\mathrm{N}$ ), plus la puissance du signal reçu est sensible à l'écart entre ${ }_{\mathrm{a}}$ et ${ }_{\mathrm{B}}$. Ainsi, dans le cas où $\mathrm{a}=100 \mathrm{~Hz}$ et ${ }_{\mathrm{B}}=99 \mathrm{~Hz}$, l'écho sera atténué respectivement de $-1 \mathrm{~dB},-5 \mathrm{~dB}$ et $40 \mathrm{~dB}$ selon que le nombre de sinusoïdes, N, est égal à 25,50 ou 100 , ce qui correspond à des durées d'impulsion sonore de $0,25 \mathrm{~s}, 0,5 \mathrm{~s}$ et $1 \mathrm{~s}$.

Puisque le Rass est destiné à effectuer des mesures sur une épaisseur étendue dans laquelle la température virtuelle varie, il est nécessaire d'émettre successivement plusieurs fréquences acoustiques afin que la condition de Bragg soit satisfaite au mieux à tous les niveaux de mesure. $\mathrm{Si}_{\mathrm{m}}$ et ${ }_{\mathrm{M}}$ désignent les fréquences de Bragg correspondant aux températures minimales et maximales susceptibles d'être observées, la suite des fréquences sonores émises est de la forme :

$\mathrm{m}, \mathrm{m}+\varepsilon, \mathrm{m}+2 \varepsilon, \quad \mathrm{m}+3 \varepsilon, \ldots \ldots, \quad \mathrm{M}$

où $\varepsilon$ représente un pas fréquentiel tel que $: \varepsilon<_{\mathrm{M}^{-}} \mathrm{m}$. Cette séquence est répétée plusieurs fois durant la mesure d'un profil de $\mathrm{T}_{\mathrm{v}}$. De plus, conformément aux résultats du paragraphe précédent, chacune de ces fréquences doit être émise pendant une durée suffisamment brève pour que la mesure de ${ }_{\text {B }}$ soit possible.

Compte tenu de ce mode de fonctionnement, la condition de Bragg à une altitude $\mathrm{z}$ donnée n'est pas identiquement respectée pour chaque émission de fréquence. La puissance moyenne de l'écho provenant de l'altitude z peut s'écrire :

$\overline{\mathrm{P}}(\mathrm{z})=\frac{1}{\mathrm{n}} \sum_{\mathrm{i}=1}^{\mathrm{n}} \mathrm{P}(\mathrm{z}, \mathrm{i})$

où $\mathrm{n}$ représente le nombre de fréquences émises durant la mesure et $\mathrm{P}(\mathrm{z}, \mathrm{i})$ la puissance de l'écho correspondant à la fréquence i telle que :

$\mathrm{i}={ }_{\mathrm{B}}+\delta_{\mathrm{i}}$

Si $\Delta$ représente l'excursion maximale de fréquence autour de ${ }_{\text {в }}$ (la valeur maximale de $|\delta|$ ), l'intensité de l'écho provenant de l'altitude z sera d'autant plus faible que $\Delta$ est important et $\mathrm{N}$ grand. Cependant, cette perte de signal sera

Figure 2 - Variations de la puissance de l'écho du Rass en fonction de l'écart entre la fréquence sonore émise et la fréquence de Bragg, pour différentes valeurs de $\mathrm{N}$ (nombre de sinusoïdes contenues dans le signal sonore). (a) $: \mathrm{N}=25 ;$ (b) $: \mathrm{N}=50$; (c) $: \mathrm{N}=100$. 


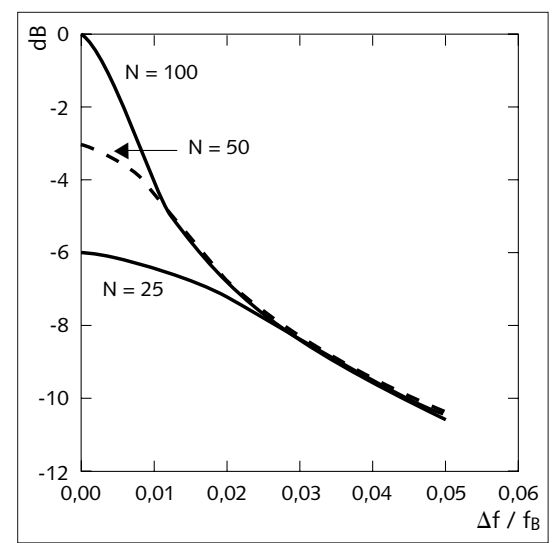

Figure 3 - Réduction de l'intensité du signal du Rass en fonction de l'excursion relative de fréquence utilisée lors d'une séquence de mesures.

\section{INFLUENCE DES CONDITIONS ATMOSPHÉRIQUES}

\author{
Atténuation \\ des ondes sonores
}

\section{Entraînement des ondes sonores par le vent}

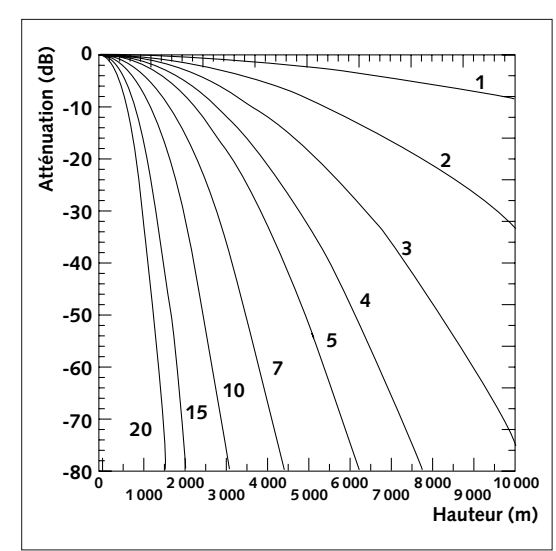

Figure 4 - Atténuation du signal du Rass due à l'entraînement des ondes sonores par un vent constant (valeurs en m. $\mathrm{s}^{-1}$ indiquées sur chaque courbe). Diamètre équivalent de l'antenne radar: $D_{e}=60 \mathrm{~m}$. en partie compensée par le fait que, d'après l'équation (2) du Rass, l'intensité de l'écho est proportionnelle à $\mathrm{N}^{2}$. Le bilan global est reporté figure 3 où la variation de puissance de l'écho Rass est représentée, pour trois valeurs de $\mathrm{N}$, en fonction de l'excursion relative de fréquence $\Delta /{ }_{\mathrm{B}}$.

Cette figure montre que, pour des valeurs faibles de cette excursion $\left(\Delta /{ }_{\mathrm{B}}<0,02\right)$, les meilleures conditions de réception sont obtenues pour des durées d'émission longues $(\mathrm{N}=100)$ alors que, pour les valeurs de $\Delta /{ }_{\text {B }}$ supérieures à 0,025 , l'intensité de l'écho Rass est indépendante de $\mathrm{N}$. On constate également que la réduction de la puissance reçue peut être significative même lorsque la gamme des fréquences émises demeure peu étendue. Ainsi, une excursion relative de $4 \%$ autour de la fréquence de Bragg se traduit par une perte de puissance de près de $10 \mathrm{~dB}$ par rapport au cas où l'accord de Bragg serait maintenu durant toute la durée de la mesure.

Toutefois, ces résultats doivent être nuancés dans la mesure où l'analyse à laquelle nous avons procédé suppose implicitement que la température virtuelle est uniforme dans une porte du radar, alors qu'en réalité elle varie. Dans ces conditions, les contraintes sur l'accord de Bragg sont moins sévères et la réduction de l'intensité de l'écho moins importante.

Les résultats précédents ont été établis en supposant une atmosphère idéale. En réalité, lors de sa propagation dans l'air, l'onde acoustique subit un certain nombre de perturbations susceptibles de modifier les performances du Rass.

Le coefficient d'atténuation du son dans l'air se décompose en plusieurs termes dont le plus important est l'atténuation moléculaire. Fonction de la température et de l'humidité, cette dernière augmente avec la fréquence sonore : de l'ordre de $10^{-2} \mathrm{~dB}$ par $100 \mathrm{~m}$ pour une fréquence de l'ordre de $100 \mathrm{~Hz}$, elle atteint quelques dB par $100 \mathrm{~m}$ aux alentours de $2000 \mathrm{~Hz}$ (Harris, 1963). En conséquence, un Rass fonctionnant avec un radar VHF sera peu affecté par ce phénomène, alors que les Rass de la bande UHF auront des portées limitées à cause de l'atténuation des ondes sonores.

Considérons un Rass où l'émetteur sonore est situé au centre $\mathrm{O}$ de l'antenne radar. En l'absence de vent, les fronts d'onde acoustiques jouent le rôle de «miroirs » sphériques focalisant l'écho du Rass en O. En revanche, sous l'effet d'un vent horizontal, l'onde acoustique s'écarte progressivement de la verticale du point d'émission au fur et à mesure de sa propagation dans l'atmosphère. Dans le cas d'une atmosphère isotherme, le déplacement $\rho$ de l'onde sonore à l'altitude z s'écrit simplement :

$\rho=\frac{1}{c_{a}} \int_{0}^{z} V(x) d x$

où $\mathrm{V}(\mathrm{x})$ est la vitesse horizontale du vent à l'altitude $\mathrm{x}$.

À cause de cet entraînement, la cible acoustique subit un déplacement à l'intérieur du faisceau radar. Les conditions de réception n'étant plus optimales, l'intensité de l'écho diminue régulièrement lorsque l'altitude augmente. À partir d'une certaine hauteur (correspondant à la portée maximale du Rass), le déplacement est tel que l'onde sonore n'est plus détectée par le radar.

L'étude de l'entraînement des ondes sonores dans le cas d'une atmosphère présentant des gradients verticaux de vitesse et de température a été effectuée par Masuda (1988). Ce dernier a montré que seules sont susceptibles de produire des échos les parties du front d'ondes dont les normales tombent au sol dans la zone occupée par l'antenne radar. En supposant que l'énergie rayonnée par l'antenne radar possède une répartition angulaire gaussienne, Lataitis (1992) trouve que l'atténuation de l'écho du Rass induite par le vent a pour expression :

$I=\exp \left[-4\left(\frac{\rho}{D_{e}}\right)^{2}\right]$

où $\mathrm{D}_{\mathrm{e}}$ est le diamètre équivalent de l'antenne radar. La figure 4 , qui représente la variation de I en fonction de l'altitude pour différentes valeurs de la vitesse 


\section{Influence de la turbulence atmosphérique}

\section{Distribution des normales}

Figure 5 - Distribution au sol des normales au front d'onde acoustique situé à l'altitude 4000 mètres. L'axe des abscisses représente les distances horizontales. L'émetteur sonore est situé au centre de l'antenne radar $(x=0)$.

(a) : atmosphère isotherme et sans vent ; (b) : vent constant de $5 \mathrm{~m} \cdot \mathrm{s}^{-1}$

(c) : profils de température $\left(-5^{\circ} \mathrm{C} \cdot \mathrm{km}^{-1}\right)$ et de vent $\left(5 \mathrm{~m} \cdot \mathrm{s}^{-1}\right)$ perturbés (écarts types: $\sigma_{\mathrm{T}}=1,5 \mathrm{~K}, \sigma_{\mathrm{v}}=1 \mathrm{~m} \cdot \mathrm{s}^{-1}$ ).

du vent, montre que l'atténuation est importante même dans le cas de vents relativement faibles. En fait, ce phénomène d'entraînement des ondes sonores par le vent constitue la cause principale limitant les performances en portée du Rass.

La turbulence atmosphérique se traduit par des fluctuations de la température et de la vitesse du vent, et donc de l'indice de réfraction acoustique de l'air. En se propageant dans un tel milieu, le front d'onde acoustique va subir des déformations locales et perdre ainsi sa cohérence spatiale. Les conséquences de ce phénomène sur le signal du Rass ont été analysées, entre autres, par Clifford et Wang (1977), Clifford et al. (1978), Bhatnagar et Peterson (1979) et Lataitis (1992). De ces différents travaux, il ressort que la présence de la turbulence atmosphérique se traduit par deux effets :

- Le premier est une atténuation du signal du Rass dépendant de la constante $C_{n}^{2}$ de structure de l'indice de réfraction acoustique de l'air.

- Le second est une perte de focalisation de l'écho du Rass. L'image au sol devient une « tache » plus ou moins étalée selon l'intensité de la turbulence. Ce phénomène peut du reste compenser partiellement l'atténuation due à l'entraînement par le vent. En effet, grâce à cet étalement, un écho qui, en l'absence de turbulence, serait situé en dehors de l'antenne, peut occuper partiellement cette dernière.

En adoptant l'approche de Masuda (1988) et en utilisant les équations de la propagation d'un rayon sonore dans l'espace (Krieble, 1971), Ouazzani Touhami (1994) a calculé la distribution au sol des normales relatives à la partie du front d'onde interceptée par le faisceau radar. Quelques-uns des résultats de cette simulation sont représentés sur la figure 5.
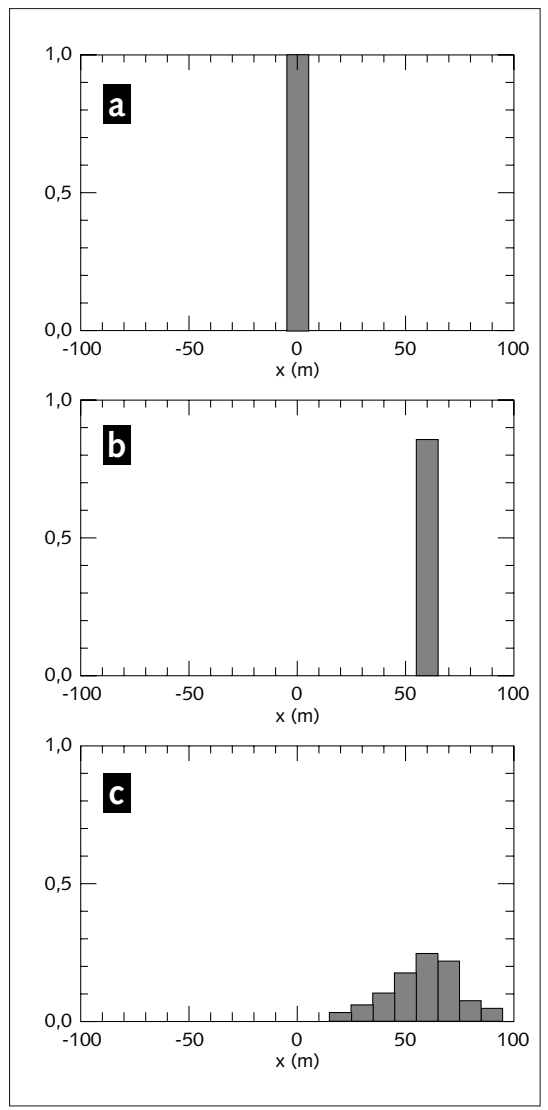

Dans une atmosphère isotherme et sans vent (figure 5a), les fronts d'onde acoustiques sont des cercles concentriques. Dans ce cas, les normales convergent au centre de l'antenne radar et les conditions de réception des échos du Rass sont donc optimales. En présence d'un vent constant avec l'altitude (figure $5 \mathrm{~b}$ ), les fronts d'onde deviennent des ellipsoïdes asymétriques ; le point de convergence des normales subit une translation et peut, si le vent est suffisamment fort, dépasser les limites de l'antenne.

Lorsque des perturbations aléatoires sont introduites sur les profils de température et de vent, la distribution des normales (figure 5c) s'étale et certaines des normales tendent à se rapprocher de l'origine, c'est-à-dire du centre de l'antenne radar. Parallèlement, l'intensité du maximum de la distribution subit une réduction importante et représente moins de $30 \%$ du cas idéal. Cette dernière simulation schématise assez bien les effets induits par la turbulence atmosphérique : étalement au sol de l'écho du Rass et compensation partielle de l'entraînement par le vent.

Afin d'évaluer les performances du Rass, May et al. (1989) ainsi que Martner et al. (1993) ont comparé plusieurs centaines de mesures de la température virtuelle obtenues à l'aide d'un Rass et par radiosondage, dans des conditions météorologiques variées. Les résultats de ces comparaisons montrent un très bon accord entre les deux méthodes employées. Lorsque les données du Rass sont 
intégrées sur une durée d'environ une heure, l'écart entre les deux types de mesures est inférieur ou égal à quelques dixièmes de degrés ; par ailleurs, l'écart type sur la mesure de $\mathrm{T}_{\mathrm{v}}$ par le Rass est de l'ordre de $1{ }^{\circ} \mathrm{C}$.

Bien que l'incidence des conditions météorologiques sur la qualité des mesures effectuées par le Rass n'ait pas été analysée, il apparaît néanmoins que la composante verticale du vent peut engendrer des erreurs sur la mesure de la température virtuelle. En effet, à une altitude donnée, la vitesse radiale $\mathrm{w}_{\mathrm{r}}$ mesurée par le radar Doppler fonctionnant en mode Rass s'écrit :

$\mathrm{w}_{\mathrm{r}}=\mathrm{w}+\mathrm{c}_{\mathrm{a}}$

où $\mathrm{w}$ représente la composante verticale du vecteur vent. Ouazzani Touhami (1994) a montré que l'erreur induite par cette composante sur la mesure de la température virtuelle a pour expression :

$\Delta \mathrm{T}_{\mathrm{v}} \approx 1,7 \mathrm{w}\left(\right.$ avec $\Delta \mathrm{T}_{\mathrm{v}}$ en ${ }^{\circ} \mathrm{C}$ et $\mathrm{w}$ en $\left.\mathrm{m} \cdot \mathrm{s}^{-1}\right)$.

La solution généralement retenue pour améliorer la précision consiste à calculer une moyenne des mesures de $T_{\mathrm{v}}$ sur une durée suffisamment longue (une à deux heures) afin que la contribution de la vitesse verticale de l'air soit minimisée. Dans le cas où une meilleure résolution temporelle est souhaitée, Angevine et al. (1994) proposent une méthode de traitement du signal du Rass qui permet de mesurer simultanément $\mathrm{w}_{\mathrm{r}}$ et $\mathrm{w}$.

En ce qui concerne la portée maximale atteinte par le Rass, Martner et al. (1993) trouvent que, dans la moitié des cas analysés, un Rass VHF fournit des échos jusqu'à environ 6000 mètres tandis qu'un Rass UHF (915 MHz) ne permet pas de mesures au-delà de 700 mètres. Comme nous l'avons indiqué précédemment, ces limitations de portée résultent de l'entraînement de l'onde sonore par le vent et également, dans le cas du Rass UHF, de l'atténuation moléculaire de l'onde sonore.

En pratique, afin de compenser, au moins partiellement, cet effet d'entraînement, plusieurs émetteurs sonores disposés à la périphérie de l'antenne radar peuvent être ajoutés à la source centrale.

DISPOSITIF EXPÉRIMENTAL

Figure 6 - Principe de l'exploitation des signaux reçus par le radar. (a) : mode profileur de vent ; (b) : mode Rass.
Les mesures par Rass que nous présentons ont été obtenues à l'aide de l'un des radars ST du réseau de recherche Insu - Météo-France (Petitdidier et al., 1990) installé à l'observatoire de Physique du globe de Clermont-Ferrand. La chaîne de traitement associée à ce radar est conçue pour mesurer des fréquences Doppler comprises entre quelques hertz et quelques dizaines de hertz. Elle n'est donc pas adaptée à la mesure de la fréquence de Bragg voisine de $100 \mathrm{~Hz}$. La solution que nous avons retenue pour utiliser néanmoins le radar en sondeur Rass consiste à décaler de $100 \mathrm{~Hz}$ le signal en sortie du récepteur en le mélangeant avec un signal sinusoïdal de référence de fréquence $100 \mathrm{~Hz}$. Le radar peut alors fonctionner selon deux modes :

- En mode habituel (profileur de vent), les signaux complexes en sortie du récepteur (figure 6a) sont de la forme :

$\mathrm{e}_{\mathrm{I}}=\mathrm{A} \cos \left(2 \pi . \mathrm{D}_{\mathrm{D}} \mathrm{t}\right) \quad \mathrm{e}_{\mathrm{R}}=\mathrm{A} \sin \left(2 \pi{ }_{\mathrm{D}} \cdot \mathrm{t}\right)$

où ${ }_{D}$ représente la fréquence Doppler associée à la vitesse radiale du vent. $D$ est directement mesurable par la chaîne de traitement numérique du radar.

a

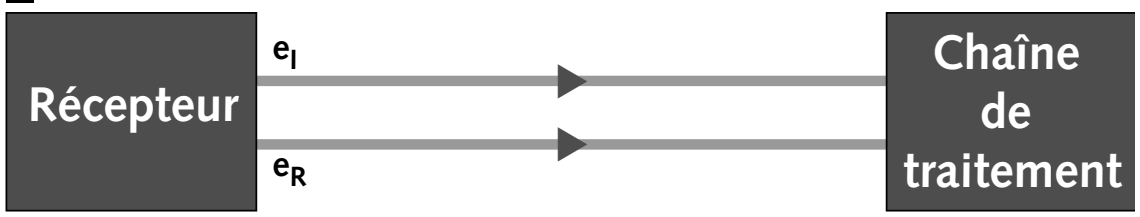

b

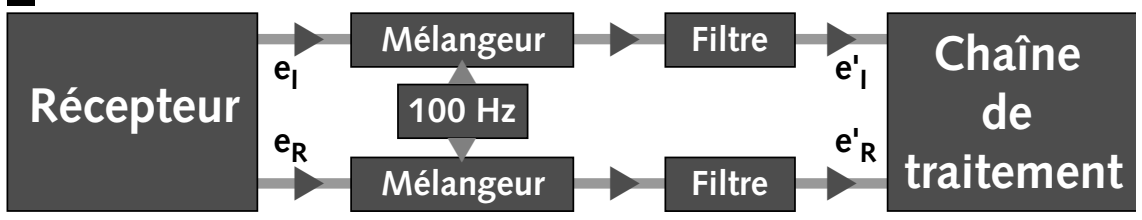


En mode Rass (figure 6b), les signaux complexes en sortie du récepteur s'écrivent :

$\mathrm{e}_{\mathrm{I}}=\mathrm{A} \cos (2 \pi$.

$\mathrm{e}_{\mathrm{R}}=\mathrm{A} \sin (2 \pi . \quad \mathrm{B} \cdot \mathrm{t})$

où ${ }_{B}$ désigne la fréquence de Bragg :

B $=100 \pm \delta$

$\delta$ étant au plus égal à quelques hertz.

Après filtrage, les signaux résultant du mélange (du produit) avec la référence $\cos (2 \pi .100 . t)$ sont de la forme :

$\mathrm{e}_{\mathrm{I}}^{\prime} \propto \cos (2 \pi . \delta . \mathrm{t}) \quad \mathrm{e}_{\mathrm{R}}^{\prime} \propto \sin (2 \pi . \delta . \mathrm{t})$

$\delta$ étant directement mesurable par la chaîne de traitement.

Ce dispositif expérimental, de conception simple, s'insère aisément dans l'équipement radar existant. Sa principale limitation tient au fait qu'il ne permet pas d'obtenir simultanément les profils de vitesse et de température virtuelle. Le passage de l'une à l'autre de ces mesures est assuré par un système de commutation à relais qui permet d'enchaîner des séquences dont la durée est de l'ordre de la minute.

Figure 7 - Schéma de principe de l'émetteur acoustique.

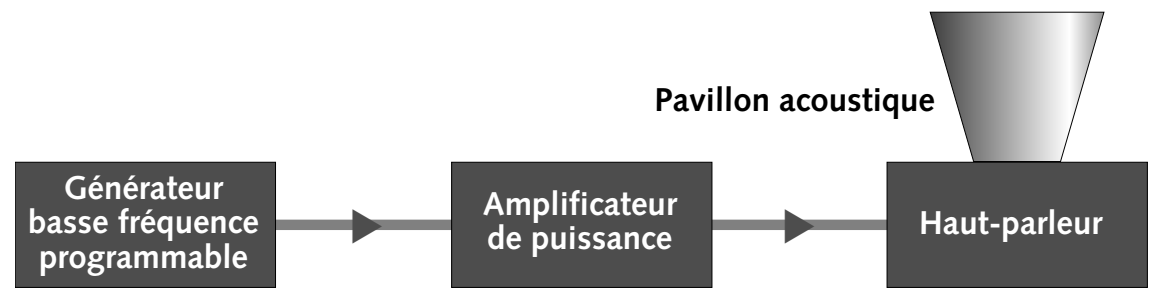

\begin{tabular}{|l|c|}
\hline Fréquence du radar $(\mathrm{MHz})$ & 45 \\
\hline Puissance crête $(\mathrm{kW})$ & 4,5 \\
\hline Durée de l'impulsion émise ( $\mu \mathrm{s})$ & 2,5 \\
\hline Dimensions de l'antenne (m) & $60 \times 60$ \\
\hline Angle d'ouverture du faisceau radar $\left(^{\circ}\right)$ & 6 \\
\hline
\end{tabular}

Tableau 3 - Caractéristiques de fonctionnement du radar de recherche Insu - Météo-France lors des sondages du Rass.

L'émetteur acoustique associé au radar (figure 7) comporte un générateur basse fréquence programmable numériquement, un amplificateur de puissance ainsi qu'un dispositif de rayonnement acoustique (haut-parleur et pavillon sonore). Le Rass étant installé en zone urbaine, la puissance acoustique émise a été limitée à une dizaine de watts afin de réduire à un niveau acceptable les nuisances sonores engendrées par le sondeur. Le générateur de fréquence offre deux possibilités d'émission. En mode séquentiel, la suite des fréquences émises est de la forme :

$\mathrm{m}, \quad \mathrm{m}+\varepsilon, \quad \mathrm{m}+2 \varepsilon, \ldots \ldots \ldots, \quad \mathrm{M}, \mathrm{m}, \mathrm{m}+\varepsilon, \ldots$

l'incrément de fréquence $\varepsilon$, de même que la durée d'un pas, étant programmable. Ce type de modulation linéaire pouvant induire des composantes parasites dans les spectres obtenus, un autre type d'émission a été prévu dans lequel la suite des fréquences émises dans l'intervalle $\left[\mathrm{m}_{\mathrm{M}}{ }_{\mathrm{M}}\right]$ est tout à fait aléatoire. Les principaux paramètres de fonctionnement du radar en mode Rass sont donnés dans le

\begin{tabular}{|l|c|}
\hline Puissance électrique (W) & 100 \\
\hline Puissance acoustique (W) & $\approx 10$ \\
\hline Dimensions du pavillon sonore (m) & $1,5 \times 1,5$ \\
\hline Angle d'ouverture $\left(^{\circ}\right)$ & $\approx 50$ \\
\hline
\end{tabular}

Tableau 4 - Caractéristiques de l'émetteur acoustique du Rass. 
tableau 3 alors que les caractéristiques de l'émetteur sonore figurent dans le tableau 4. Tous les sondages par Rass ont été effectués avec la source sonore placée au centre de l'antenne du radar ST. Les fréquences émises couvraient la gamme comprise entre ${ }_{\mathrm{m}} \approx 95 \mathrm{~Hz}$ et ${ }_{\mathrm{M}} \approx 103 \mathrm{~Hz}$, l'incrément de fréquence étant le plus souvent égal à $0,2 \mathrm{~Hz}$. La résolution temporelle des mesures de température était, en général, de 2 à 3 minutes.

\section{RÉSULTATS}

La figure 8 représente des spectres typiques d'échos de Rass obtenus à l'aide du dispositif expérimental décrit précédemment. Dans cet exemple, le signal associé au Rass apparaît nettement entre les niveaux 2,6 km et $6 \mathrm{~km}$. Le décalage régulier de ce signal vers la gauche traduit la décroissance progressive de la température virtuelle avec l'altitude. À partir de tels spectres, la fréquence de Bragg associée à l'écho du Rass est déduite pour chaque porte de mesure, puis la température virtuelle correspondante est calculée à l'aide de la relation (8).

Quatre expériences ont été effectuées, dans lesquelles le profil vertical de la température virtuelle a été mesuré simultanément par radiosondage et à l'aide du Rass. Ces expériences se sont déroulées en présence de conditions météorologiques non perturbées (ciel clair à peu nuageux, vent faible). Afin de minimiser les erreurs induites par la composante verticale du vent, une moyenne des données du Rass a été effectuée à chaque niveau de mesure sur une période de deux heures encadrant l'instant du début du radiosondage.

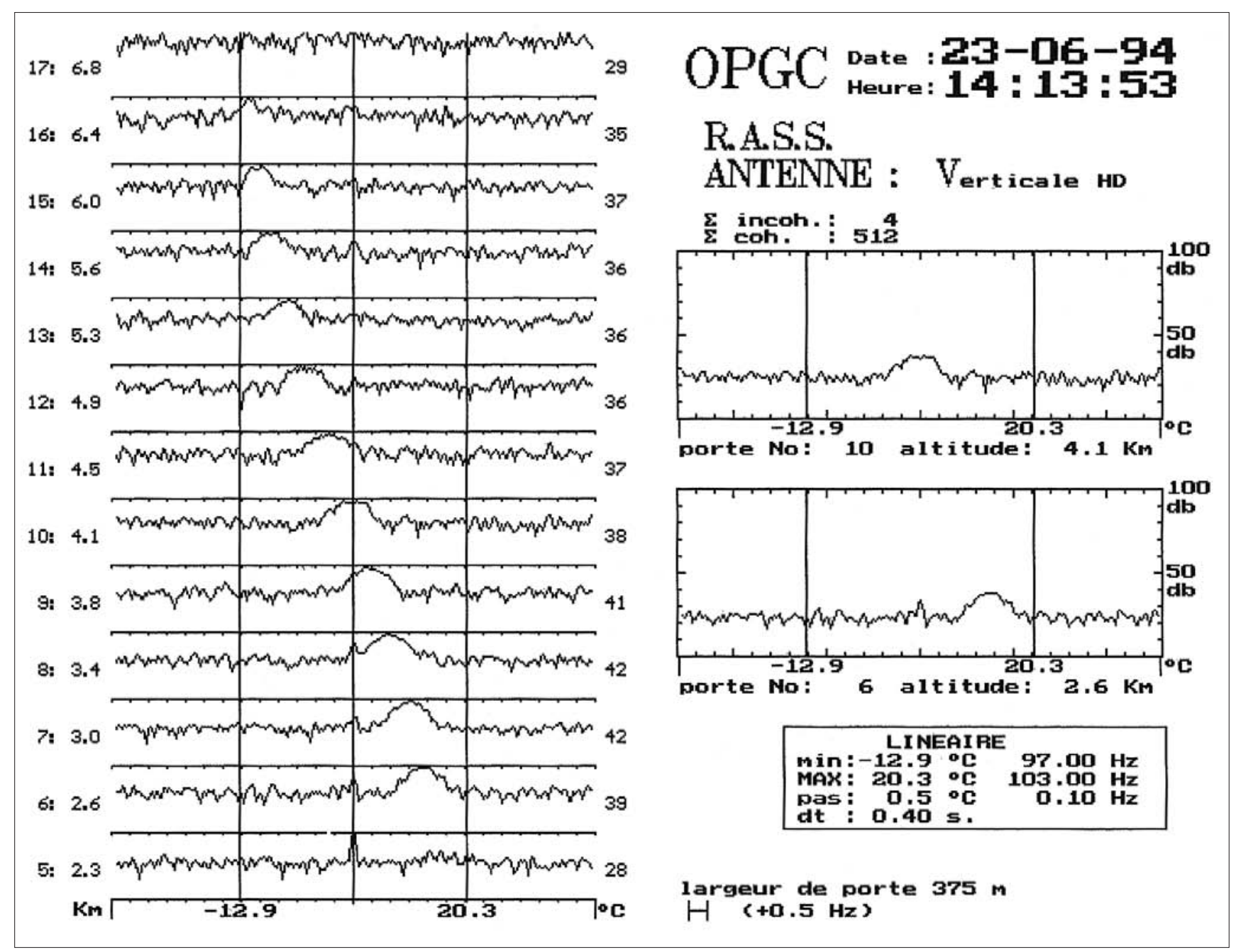

Figure 8 - Exemple de spectres d'échos du Rass. Chaque spectre correspond à une porte de mesure dont le numéro est donné dans la colonne de gauche et l'altitude dans la colonne suivante. Pour chaque spectre, la fréquence de Bragg est donnée par l'abscisse moyenne du signal du Rass. Les deux graphes de droite sont des agrandissements des portes 6 et 10. Les données encadrées, en bas à droite, représentent les conditions de fonctionnement de l'émetteur acoustique. 
Les résultats de ces quatre séries de comparaisons (figures 9a-d) montrent que les mesures de la température virtuelle fournies par le Rass sont en bon accord avec celles du radiosondage.

En considérant, pour ces quatre expériences, l'ensemble des mesures brutes fournies par le Rass $\left(\mathrm{T}_{\mathrm{v} \text { Rass }}\right.$ ) sur le même intervalle de temps de deux heures, on trouve également (figure 10) un accord satisfaisant avec les données du radiosondage $\left(\mathrm{T}_{\mathrm{v} \text { Radio }}\right)$. Pour les 250 données du Rass utilisées, la valeur moyenne de la différence $\left(\mathrm{T}_{\mathrm{v} \text { Rass }}-\mathrm{T}_{\mathrm{v} \text { Radio }}\right)$ est égale à $0,07{ }^{\circ} \mathrm{C}$, l'écart type correspondant valant $0,85{ }^{\circ} \mathrm{C}$. Ces valeurs sont conformes aux résultats des comparaisons effectuées par Martner et al. (1993) avec un Rass VHF.

Comme le profileur de vent, le Rass ne fournit pas de mesures en dessous de 2000 mètres. Il s'agit là d'une limitation propre à tous les radars ST VHF. Pour obtenir des mesures de la température virtuelle dans les basses couches de l'atmosphère, il est nécessaire d'utiliser un Rass fonctionnant dans la gamme UHF (voir tableau 2). En ce qui concerne la portée maximale atteinte par le Rass, celle-ci se situe généralement aux alentours de 4000 mètres (cas des figures $9 \mathrm{a}$, $9 \mathrm{c}$ et $9 \mathrm{~d})$. Beaucoup plus rarement, des échos peuvent être détectés jusqu'à envron 6000 mètres (exemple de la figure 9b). Nous avons vu précédemment

\begin{tabular}{|c|c|c|}
\hline & $\mathrm{Z}_{\mathrm{P}}(\mathrm{m})$ & $\mathrm{Z}_{\mathrm{T}}(\mathrm{m})$ \\
\hline 16 juin 1994 & 3500 & 3200 \\
\hline 23 juin 1994 & 6000 & 6800 \\
\hline 12 juillet 1994 & 4000 & 3300 \\
\hline 30 novembre 1994 & 4000 & 4300 \\
\hline
\end{tabular}

Tableau 5 - Portée maximale du Rass déterminée à partir des profils de la température virtuelle $\left(Z_{P}\right)$ et à partir de la trajectoire de l'onde sonore $\left(Z_{T}\right)$.
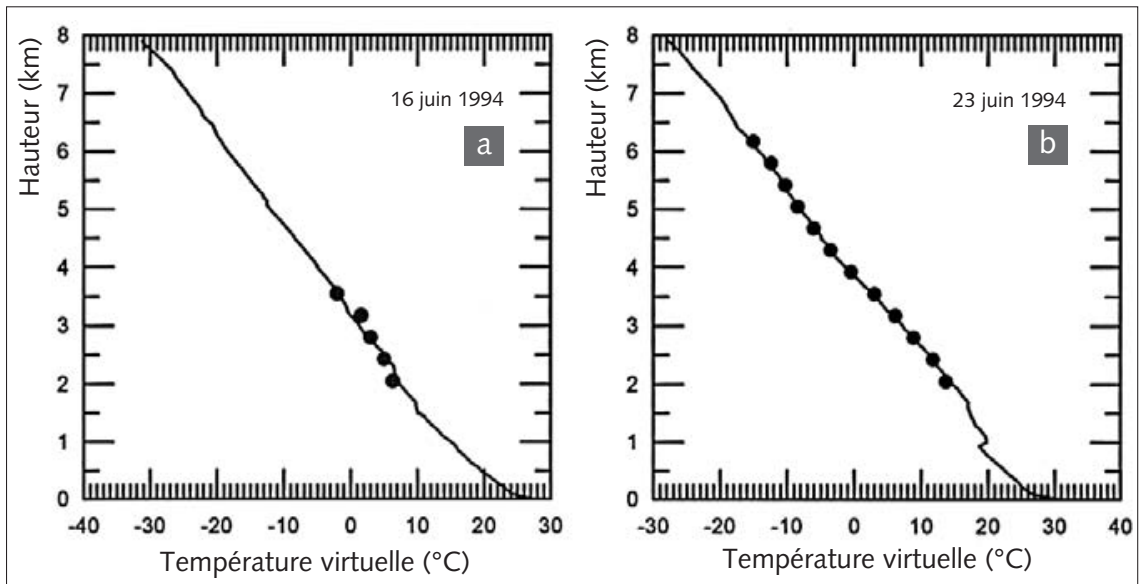

Figure 9 - Exemples de profils de la température virtuelle $T_{v}$ obtenus à l'aide du Rass (points) et par radiosondage (courbe) pour quatre journées de vent faible ou très faible. Chaque point représente une moyenne des mesures du Rass sur une période de deux heures.
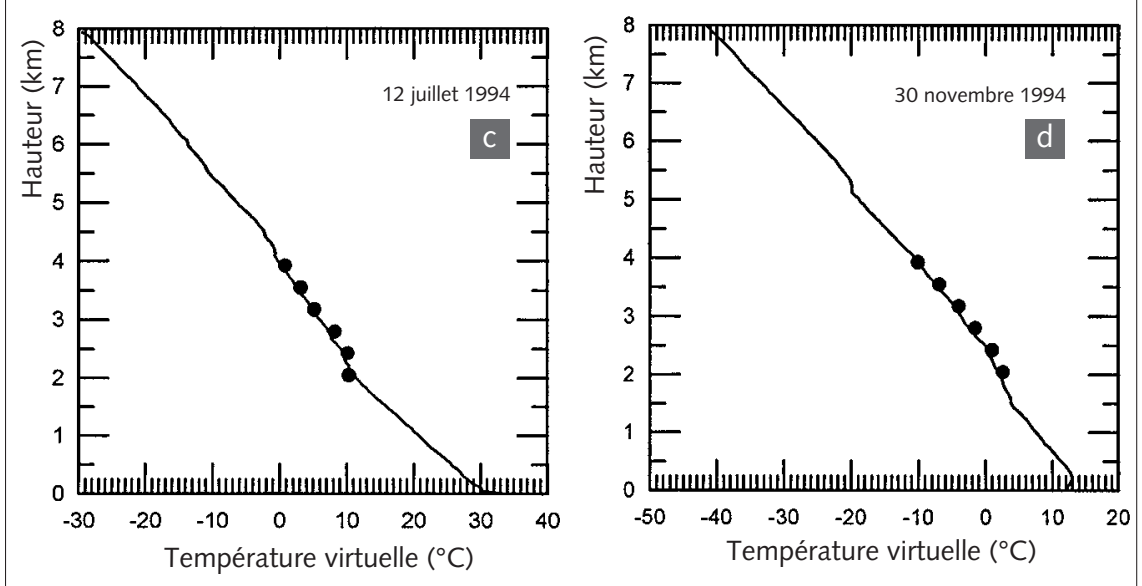


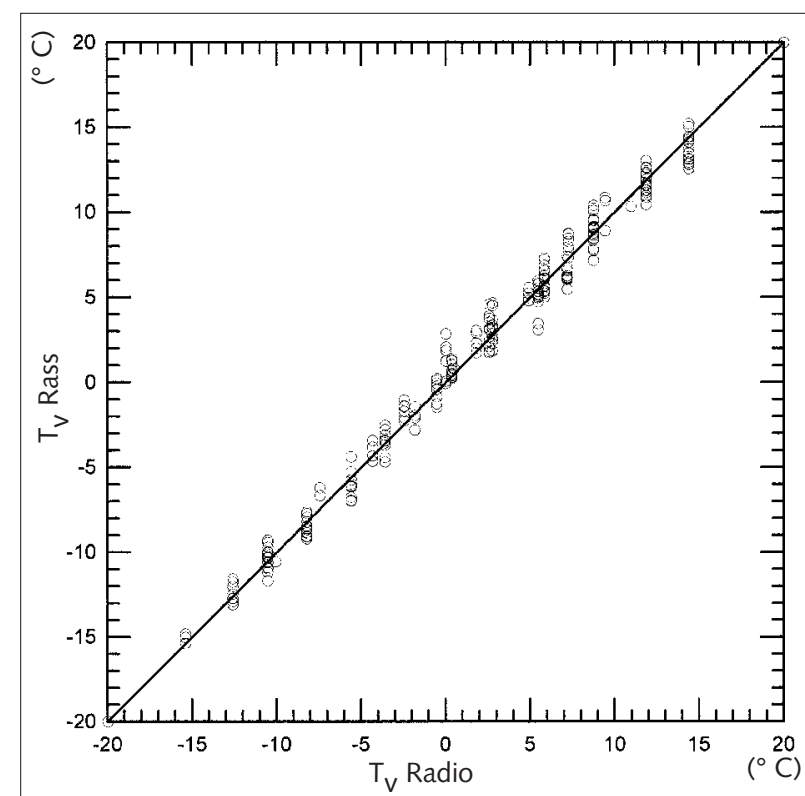

Figure 10 - Comparaison entre les mesures de la température virtuelle réalisées à l'aide du Rass (données brutes) et par radiosondage.

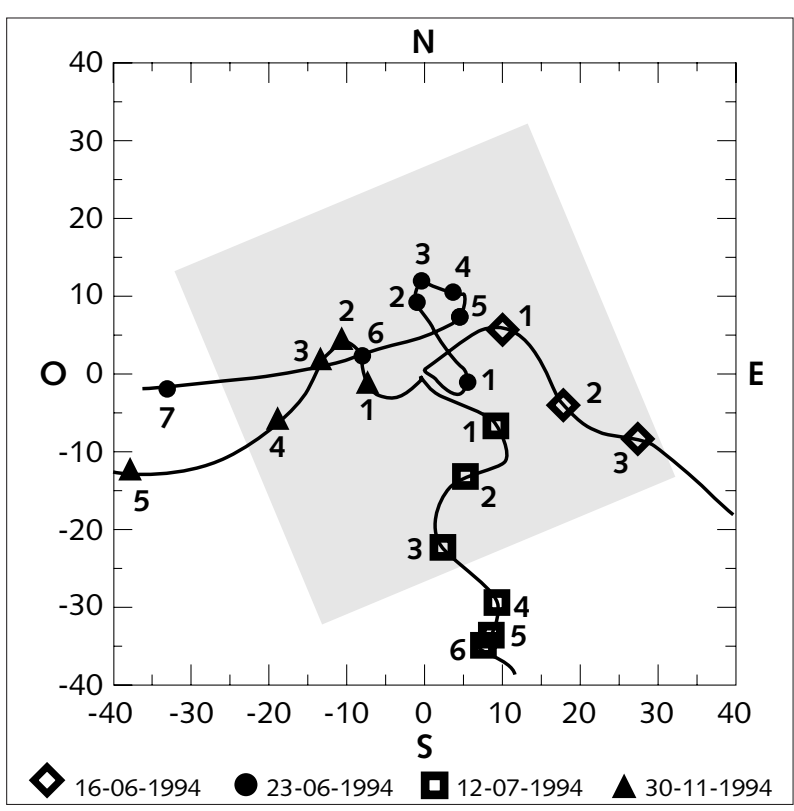

que cette limitation de portée est due principalement à l'entraînement des ondes sonores par le vent. Selon l'approche de Masuda (1988), cette situation correspond au cas où les normales au front d'onde acoustique tombent au sol en dehors de l'antenne radar. En admettant, en première approximation, que l'onde sonore est sphérique, l'ensemble des normales à cette onde, à une altitude donnée, convergent au sol en un point unique $\mathrm{C}$ dont la position peut être calculée à l'aide de la relation (10). La portée maximale du Rass, $\mathrm{Z}_{\mathrm{T}}$, correspond approximativement à l'altitude pour laquelle la trajectoire de $\mathrm{C}$ coupe la limite de l'antenne du radar. En utilisant le profil de vent mesuré par radiosondage, nous avons déterminé cette trajectoire pour les quatre journées considérées (figure 11) ainsi que les valeurs de $\mathrm{Z}_{\mathrm{T}}$ correspondantes. Le tableau 5 montre que ces dernières sont assez proches de celles déduites des figures $9 \mathrm{a}-\mathrm{d}$. Les écarts observés ont probablement plusieurs causes. D'une part, dans une atmosphère non isotherme et en présence de vent, les ondes sonores se propagent sous la forme d'ellipsoïdes et non, comme nous l'avons supposé, de sphères. Par ailleurs, l'effet de la turbulence sur la distribution au sol des normales n'a pas été pris en compte. Enfin, du point de vue radioélectrique, l'antenne radar ne peut être assimilée à sa surface géométrique.

Comme nous l'avons signalé, l'utilisation de plusieurs sources sonores disposées autour de l'antenne du radar permet de compenser, en partie, l'effet d'entraînement dû au vent. Une autre solution consiste à utiliser un émetteur acoustique mobile placé de telle sorte que l'onde qu'il émet, entraînée par le vent, se trouve à la verticale de l'antenne pour des altitudes élevées. Pour cette dernière configuration, des courbes telles que celles de la figure 11 permettent de localiser le point d'émission sonore le mieux adapté. En l'absence de radiosondage, la trajectoire de l'onde sonore peut être calculée en temps réel à partir des profils de vent obtenus à l'aide du radar ST. Toutefois, dans ce cas, en raison de l'absence de mesures en dessous de l'altitude 2000 mètres, la trajectoire de l'onde sonore ainsi que la position du point d'émission sont connues avec une précision moindre.

Figure 11 - Projection au sol de la trajectoire de l'onde sonore reconstituée à partir du profil de vent obtenu par radiosondage. Pour une courbe donnée, le nombre figurant à côté de chaque symbole représente l'altitude correspondante $(\mathrm{km})$. Le carré en grisé au centre de la figure matérialise le contour de l'antenne du radar. Les échelles des deux axes sont en mètres.
CONCLUSION

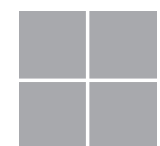

Longtemps cantonné à la seule couche limite planétaire, le domaine d'utilisation du Rass s'étend désormais à une grande partie de la troposphère. Ces progrès substantiels ont pu être accomplis grâce à l'utilisation de radars ST, dont les performances sont bien supérieures à celles des dispositifs jusqu'alors utilisés pour les sondages radioacoustiques de l'atmosphère. L'association Rass-profileur de vent présente, en outre, l'avantage de fournir simultanément des mesures de vent et de température. À cet égard, les études sur les fronts réalisées par Neiman et al. (1991 et 1992) montrent tout le profit que l'on peut tirer d'un tel dispositif de sondage.

Les résultats que nous avons obtenus avec l'un des radars $45 \mathrm{MHz}$ du réseau de recherche Insu - Météo-France sont encourageants. En effet, moyennant quelques modifications de la chaîne de réception ainsi que la mise en place d'un émetteur sonore simple et peu coûteux, ce profileur est capable de fournir, en configuration Rass, des profils verticaux de la température virtuelle avec une bonne précision. Les performances en portée, quelque peu limitées, pourraient 
être améliorées en augmentant la puissance acoustique émise et en utilisant plusieurs sources acoustiques (ou une source mobile) afin de compenser l'entraînement des ondes sonores par le vent. Enfin, la mise en service prochaine sur les radars du réseau de recherche d'une nouvelle chaîne de traitement permettra une utilisation simultanée des modes profileurs de vent et de température.

$\mathrm{Si}$, au cours des dernières années, les sondages radioacoustiques de l'atmosphère ont connu des progrès substantiels, le problème posé par l'entraînement de la cible sonore hors du faisceau du radar dans des conditions de vent fort n'a pas été, à ce jour, résolu de manière véritablement satisfaisante. Néanmoins, sans pouvoir rivaliser avec les performances atteintes par le radiosondage (particulièrement en ce qui concerne la portée verticale et la résolution spatiale), le Rass fournit des mesures précises de la température virtuelle avec une bonne résolution temporelle.

\section{Remerciements}

BIBLIOGRAPHIE
Le sondeur Rass a été développé par R. Cordesses et l'équipe technique de l'OPGC ( C. Hervier, V. Kromer, G. Michel et M. Rahon). Les radiosondages ont été effectués par R. Hervier (Lamp-OPGC) à l'aide de la station mise à notre disposition par le CNRM. Le réseau des radars Insu - Météo-France est financé conjointement par l'Insu (CNRS) et par Météo-France.
Adachi T., T. Tsuda, Y. Masuda, T. Takami, S. Kato et S. Fukao, 1992 : Effects of the acoustic and radar pulse length ratio on the accuracy of radio acoustic sounding system (RASS) temperature measurements with monochromatic acoustic pulses. Radio Sci., 28, 571-583.

Angevine W. M., W. Ecklund, D. A. Carter et K. S. Gage, 1994 : Improved radio acoustic sounding techniques. J. Atmos. Oceanic Technol., 11, 42-49.

Atlas D., 1962 : Indirect probing technique. Bull. Am. Meteorol. Soc., 43, 457-466.

Bhatnagar N. et A. M. Peterson, 1979 : Interaction of electromagnetic and acoustic waves in a stochastic atmosphere. IEEE Trans. Antennas propag., AP-27, 385-393.

Clifford S. F. et T. Wang, 1977 : The range limitation on radar acoustic sounding system (RASS) due to atmospheric refractive turbulence. IEEE Trans. Antennas propag., AP-15, 319-326.

Clifford S. F., T. Wang et J. T. Priestly, 1978 : Spot size of the radar return from a radar acoustic sounding system (RASS) due to atmospheric refractive turbulence. Radio Sci., 13, 985-989.

Fetter R. W., 1961 : The EMAC probe - a new concept for atmospheric sounding by radar. Proc. 9th Weather Radar Conference, Kansas City, 385-390.

Frankel M. S. et A. M. Peterson, 1976 : Remote temperature profiling in the lower troposphere. Radio Sci., 11, 157-166.

Harris C. M., 1963 : Absorption of sound in air in the audio-frequency range. J. Acoust. Soc. Am., 35, 11-17.

Klaus V., J. Pilon, G. Chérel et J.-M. Donier, 1994 : La mesure automatique du vent en altitude. Le profileur de vent en phase préopérationnelle. La Météorologie 8 série, 7, 6-24.

Kon A. I. et V. I. Tatarskii, 1980 : The scattered signal frequency spectrum for radioacoustical atmospheric sounding. Isz. Acad. Sci. USSR Atmos. Oceanic Phys., Engl. Transl., 16, 142-148.

Krieble A. R., 1971 : Refraction and attenuation of sound by wind and thermal profiles over a ground plane. J. Acoust. Soc. Am., 51(1), 19-23.

Lataitis R. J., 1992 : Signal power for radio acoustic sounding of temperature: the effects of horizontal winds, turbulence and temperature gradient. Radio Sci., 27, 369-385.

Marshall J. M., 1970 : A radio acoustic sounding system for the remote measurement of atmospheric parameters. Stanford Electron. Lab., Stanford, Californie, États-Unis, SEL-70-050, 119 p.

Martner B. E., D. B. Wuertz, B. B. Stankov, R. G. Strauch, E. R. Westwater, K. S. Gage, W. L. Ecklund, C. L. Martin et W. F. Dabberdt, 1993 : An evaluation of wind profiler, RASS, and microwave radiometer performances. Bull. Am. Meteorol. Soc., 74, 599-613. 
Masuda Y, 1988 : Influence of wind and temperature on the height limit of a radio acoustic sounding system. Radio Sci., 23, 647-655.

May P. T., K. P. Moran et R. G. Strauch, 1989 : The accuracy of RASS temperature measurements. J. Appl. Meteor., 28, 1329-1335.

May P. T., R. G. Strauch, K. P. Moran et W. L. Ecklund, 1990 : Temperature sounding by RASS with profiler radars: A preliminary study. IEEE Trans. Geosci. Remote Sens., 28, 19-28.

Moran K. P., D. B. Wuertz et R. G. Strauch, 1991 : Temperature sounding with wind profiler radars. J. Atmos. Oceanic Technol., 8, 606-608.

Neiman P. J., P. T. May, B. B. Stankov et M. A. Shapiro, 1991 : Radio acoustic sounding system observations of an arctic front. J. Appl. Meteor., 30, 881-892.

Neiman P. J., P. T. May et M. A. Shapiro, 1992 : Radio acoustic sounding system (RASS) and wind profiler observations of lower and midtropospheric weather systems. Mon. Wea. Rev., 120, 2298-2313.

Ouazzani Touhami T., 1994 : Mesure des profils de température par télédétection radioacoustique : étude théorique et expérimentale. Thèse de doctorat de l'université Blaise Pascal, Clermont-Ferrand.

Peters G., H. Timmermann et H. Hinzpeter, 1983 : Temperature sounding in the planetary boundary layer by RASS system: analysis and results. Int. J. Remote Sens., 4, 49-63.

Petitdidier M., V. Klaus, F. Baudin, C. Bourdier, M. Crochet, A. Desautez, C. Guerin, R. Ney, G. Penazzi et P. Quinty, 1990 : The 961/45 MHz bifrequency INSU/METEO Stratospheric-Tropospheric radar. Meteor. Rdsch., 42, 142-151.

Strauch R. G., K. P. Moran, P. T. May, A. J. Bedard Jr et W. L. Ecklund, 1989 : Rass temperature soundings with wind profiler radars. Preprints of the 24th Conference on radar meteorology, 741-745.

Tatarskii V. I., 1971 : The effects of the turbulent atmosphere on wave propagation. Israel Program for Scientific Translations, Jérusalem, $472 \mathrm{p}$.

Tsuda T., Y. Masuda, H. Inuki, K. Takahashi, T. Takami, T. Sato, S. Fukao et S. Kato, 1989 : High time resolution monitoring of tropospheric temperature with a radio acoustic sounding system. Pageoph., 130, 497-507. 